\title{
ISOLATED BINARIES AND TRIPLES IN N-BODY SYSTEMS
}

\author{
J. ANOSOVA, \\ National Astronomical Observatory, Tokyo 181, Japan \\ AND
}

L. KISELEVA, Institute of Astronomy, Cambridge CB3 OHA, UK

Stars show a marked tendency to form the systems of different multiplicity starting from the smallest systems as binary and triple stars up to clusters with a significantly larger number of objects $\left(N \sim 10^{7}\right.$ for globular cluster $\mathbf{s}$ ). Different investigators have used different methods of binary stars identificati on but modern observations give a frequency of binary and multiple stars in the Galactic field up to $70 \%$ Binary and multiple stars are also often present within star clusters. It is therefore very important to be able to identify such systems as rather isolated substructures in which the dynamical evolution is not significantly affected by other stars (at least, during some considerab le time interval).

The character of basic Newtonian regular and chaotic motions of the bodies inside triple and $N>3$-body stellar systems was studied using computer simulations. It is shown that in many cases the basic motions of the bodies in both types of systems are similar. In the clusters one can observe roughly superposition of motions of singular bodies as well as the different types of weak and strong two- and three-body interactions. One may therefore study in detail the interactions of the bodies in the $\mathrm{N}$-body systems by means of computer simulations in the framework of the isolated general three-body problem. It is necessary to distinguish the regular and irregular fields in the clusters and also to consider representative samples of initial conditions for triples with both positive and negative total energies. Different criteria of isolation of subsystems within clusters as well as multiples and clusters in the general field of stars and galaxies are discussed and the new dynamical criteria of isolation of binaries and multiples are developed. 